\title{
RANDOM WALKS, ELLIPTIC INTEGRALS AND RELATED CONSTANTS
}

\author{
JAMES GU FENG WAN
}

(Received 12 September 2013; first published online 26 November 2013)

2010 Mathematics subject classification: primary 33C20; secondary 33C05, 33C45, 33E05, 33F10.

Keywords and phrases: hypergeometric function, complete elliptic integral, random walk, experimental mathematics.

In the first part of this dissertation, we investigate the problem of how far a walker travels after $n$ unit steps, each taken along a uniformly random direction; the shortstep behaviour of this random walk was unknown. Utilising functional equations, we fully analyse the three- and four-step walks, finding the moments and densities of the distance from the origin. Our methods involve a blend of combinatorics, probability and complex analysis.

The derivatives of random walk moments turn out to be Mahler measures. We fruitfully study them using elementary techniques (different from those used by other researchers), namely generating functions of log-sine integrals and trigonometry. On the other hand, some random walk moments can be written as moments of products of complete elliptic integrals. These are studied, culminating in a complete solution for the moments of the product of two elliptic integrals. We also give some results when more elliptic integrals are involved. These endeavours occupy the second part of this dissertation.

A spectacular application of elliptic integrals is their ability to produce rational series which converge to $1 / \pi$, as observed by Ramanujan. Using modular forms and hypergeometric transforms, we produce new classes of $1 / \pi$ series which involve Legendre polynomials and Apéry-like sequences. We give a diverse range of series for related constants, including some based on Legendre's relation. The third part of this dissertation is devoted to this topic.

In the final part we apply experimental methods to better understand a number of areas encountered in our prior investigations. We simplify proofs for some multiple zeta value identities, give new ones and outline how they may be found. We give a

Thesis submitted to The University of Newcastle, New South Wales; degree approved September 2013; principal supervisor: Laureate Professor Jonathan M. Borwein; co-supervisor: Professor Wadim Zudilin.

(C) 2013 Australian Mathematical Publishing Association Inc. 0004-9727/2013 \$16.00 
method to quickly generate contiguous relations for hypergeometric series. Lastly, we look at orthogonal polynomials, in particular a new application of Gaussian quadrature to multi-dimensional lattice sums.

JAMES GU FENG WAN, School of Mathematical and Physical Sciences, The University of Newcastle, Callaghan, NSW 2308, Australia e-mail: james.g.wan@gmail.com 